\author{
(с) Соркина Е.Л., Тюльпаков А.Н.
}

ФГБУ «Национальный медицинский исследовательский центр эндокринологии» Минздрава России, Москва

Синдромы липодистрофии представляют собой гетерогенную группу наследственных или приобретенных редких заболеваний, характеризующихся полной (генерализованная липодистрофия) или частичной (парциальная липодистрофия) потерей подкожной жировой клетчатки, как правило, сопровождающихся различными метаболическими нарушениями. По этиологии различают наследственные и приобретенные липодистрофии. Значительный прогресс в области молекулярной генетики позволил за последние 20 лет идентифицировать 20 генов, ассоциированных с развитием различных синдромов липодистрофий. Однако, по данным большинства исследователей, примерно у половины пациентов с липодистрофией мутации в этих генах не выявляются, что предполагает необходимость, с одной стороны, дальнейших исследований в области молекулярной генетики, а с другой - исследования аутоиммунных факторов в этиологии синдромов липодистрофии.

КЛЮЧЕВЫЕ СЛОВА: липодистрофия, парциальная, генерализованная, прогероидный синдром, синдромы липодистрофий, адипогенез, аутоиммунные механизмы.

\title{
INHERITED AND ACQUIRED LIPODYSTROPHIES: MOLECULAR-GENETIC AND AUTOIMMUNE MECHANISMS
}

\author{
(c) Sorkina E.L., Tyulpakov A.N.
}

Endocrinology research centre, Moscow, Russia

Lipodystrophy syndromes form a heterogenous group of inherited or acquired rare disorders, characterized by total (generalized lipodystrophy) or partial fat loss (partial lipodystrophy), usually accompanied by different metabolic disorders. Based on etiology lipodystrophies can be inherited or acquired. As a result of a significant progress in molecular genetics 20 new genes, associated with different lipodystrophy syndromes, were discovered during the last 20 years. However according to the majority of researchers' data mutations in these causative genes are not found in approximately half of the patients. This might mean the need for both further molecular-genetic studies and the search for autoimmune factors playing a role in lipodystrophy syndromes etiology.

KEYWORDS: lipodystrophy, partial, generalized, progeroid syndrome, lipodystrophy syndromes, adipogenesis, autoimmune mechanisms.

\section{ВВЕДЕНИЕ}

Синдромы липодистрофии представляют собой гетерогенную группу наследственных или приобретенных редких заболеваний, характеризующихся полной (генерализованная липодистрофия) или частичной (парциальная липодистрофия) потерей подкожной жировой клетчатки. В литературе имеются данные о более 1000 наблюдений, распространенность данной группы заболеваний оценивается как 1:1 000000 населения [1]. Наследственные липодистрофии (как генерализованные, так и парциальные), а также приобретенные генерализованные липодистрофии, как правило, сопровождаются различными метаболическими нарушениями, такими, как сахарный диабет (СД), дислипидемия и стеатогепатит [2].

\section{КЛАССИФИКАЦИЯ}

По этиологии различают наследственные и приобретенные липодистрофии. Среди наследственных липодистрофий выделяют 3 основные группы: врожденные генерализованные липодистрофии (ВГЛ), семейные пар- циальные липодистрофии (СПЛ) и липодистрофии в рамках многокомпонентных прогероидных синдромов. Следует отметить, что большинство ВГЛ и прогероидных синдромов наследуется по аутосомно-рецессивному механизму (АР), а СПЛ - по аутосомно-доминантному (АД). Патогенез наследственных форм липодистрофий связан с нарушением адипогенеза на разных уровнях (табл. 1).

Приобретенные липодистрофии по степени потери подкожно-жировой клетчатки разделяют на генерализованные (ПГЛ) и парциальные (ППЛ). Аутоиммунные заболевания, такие как ювенильный дерматомиозит и аутоиммунный гепатит, часто сочетаются с ПГЛ, что свидетельствует о том, что ПГЛ может сама являться аутоиммунным заболеванием, но патогенетические основы этого состояния еще не изучены. У пациентов с панникулит-ассоциированной липодистрофией в сочетании с контрактурами суставов, атрофией мышц и микроцитарной анемией были выявлены гомозиготные мутации в гене PSMB8 [33].

Так же, как и ПГЛ, ППЛ наблюдается при некоторых аутоиммунных заболеваниях, таких, как дерматомиозит и системная красная волчанка. У большинства пациентов с ППЛ низкие уровни сывороточного комплемента 3 
Таблица 1. Классификация ВГЛ, СПЛ и прогероидных синдромов. Гены, ассоциированные с развитием наследственных липодистрофий

\section{Тип Мутантный ген и кодируемый белок}

Распространенность, количество описанных случаев, механизм наследования (АР/АД)

\section{ВГЛ 1}

AGPAT2, фермент 1-ацилглицерол-3-фос- Самый распространенный вариант ВГл, фат-О-ацилтрансфераза 2 [3]

\section{ВГЛ 2}

ВГЛ 3

ВГЛ 4

СПЛ 1 (синдром Кобберлинга)

СПЛ 2 (синдром Даннигана)

СПЛ 3 (синдром Даннигана)

СПЛ 4

СПл 5

СПЛ 6

СПЛ 7

Истинная прогерия Хатчинсона-Гилфорда

Синдром Вернера (прогерия взрослых)

Атипичная прогерия, атипичный синдром Вернера

Мандибуло-акральная дисплазия (МАД)

Мандибулярная гипоплазия, глухота и прогероидный синдром

Прогероидный синдром

\section{Синдром Нестера-Гильермо}

Неонатальный синдром Марфана с прогероидным синдромом

Синдром Кеппена-Любински
$B S C L 2$, белок сейпин [4]

CAV1, белок кавеолин 1 типа [5] PTRF, белок кавин [7]

Неизвестен

LMNA, ламин А и С [11]

PPARG, PPARY [12]

PLIN1, перилипин 1

CIDEC, эффектор C, подобный фактору фрагментации ДНК, индуцирующему клеточную гибель

LIPE, гормончувствительная липаза AKT2, протеинкиназа В

LMNA, ламин A

WRN, ДНК-хеликаза [19, 20]

LMNA, ламин A $[22,23]$

МАД типа A - LMNA [25], МАД типа B ZMPSTE24 [26]

$P O L D 1$, каталитическая субъединица гена полимеразы дельта 1 [28]

SPRTN, белок SprT-подобного N-терминального домена [29]

BANF1, фактор-1-барьера аутоинтеграции [30]

FBN1, фибриллин 1 [31]

KCNJ6, гетерозиготные мутации de novo 3 случая в 3 семьях, все мужского пола, [32]
AP

2-й по распространенности вариант, АР

1 случай, АР [6]

Около 20 случаев, АР $[8,9]$

Описана только у женщин [10], неизвестен

Около 300 случаев, АД

Около 30 случаев, АД

6 случаев в 3 семьях, АД [13]

1 случай, AP [14]

6 пациентов из 2 семей, AP $[15,16]$

4 пациента из 1 семьи, АД [17]

Не более 100 случаев, АР (редко), в основном мутации de novo [18]

Около 200 случаев, АР [21]

41 случай [24], АД, de novo

AP [27]

АД, de novo

3 случая в 2 семьях, AP

2 случая в 2 семьях, AP

1 случай, AP

АД
(С3) и определяются циркулирующие антитела к нему, С3-нефритический фактор. ППЛ также чаще встречается у женщин, чем у мужчин (в соотношении около 4:1) [34]. Хотя ППЛ принято относить к приобретенным липодистрофиям, основываясь на позднем дебюте заболевания и отсутствию наследственной предрасположенности, в 2006 г. Hegele et al. обнаружили мутации в гене LMNB2 у 4 пациентов с ППЛ [35]. У всех описанных пациентов были нормальные уровни С3 и показатели функции почек, не выявлялся С3-нефритический фактор, 3 имели СД, 2 - синдром поликистозных яичников (СПЯ), 1 пациент страдал дерматомиозитом и алопецией.

\section{МЕТАБОЛИЧЕСКИЕ ОСЛОЖНЕНИЯ}

Метаболические осложнения синдромов липодистрофий являются основной причиной заболеваемо- сти и смертности у данных пациентов. Дефицит жировой ткани приводит к недостаточности лептина, провоцирующей повышение аппетита и эктопическое отложение жировой ткани, что, в свою очередь, способствует инсулинорезистентности, ассоциированной с СД, гипертриглицеридемией, неалкогольной жировой болезнью печени, СПЯ, черным акантозом и ранним атеросклерозом. При большинстве форм заболевания следует проводить ежегодный скрининг на СД, дислипидемию, заболевания печени, почек и сердца. Основными причинами смертности пациентов с синдромами липодистрофий являются сердечно-сосудистые заболевания (кардиомиопатия, сердечная недостаточность, инфаркт миокарда, аритмия), заболевания печени (печеночная недостаточность, гастроинтестинальное кровотечение, гепатоцеллюлярная карцинома), почечная недостаточность, острый панкреатит и сепсис $[2,36]$. 


\section{ЛЕЧЕНИЕ}

Диета является необходимой основой лечения и профилактики метаболических осложнений при липодистрофиях. Терапия метрелептином эффективна для лечения метаболических осложнений у пациентов с генерализованной липодистрофией и низким уровнем лептина, а также у некоторых пациентов с семейной парциальной липодистрофией. Для симптоматического лечения метаболических осложнений также можно применять другие препараты, например, метформин для лечения СД, статины и фибраты для лечения дислипидемии. Противопоказан прием оральных препаратов эстрогенов [2].

\section{ЗАКЛЮЧЕНИЕ}

За последние годы наблюдается значительный прогресс в изучении этиологии как наследственных, так и приобретенных форм липодистрофий, однако многие вопросы еще требуют уточнения. Помимо дальнейших исследований в области молекулярной генетики, целесообразно изучение аутоиммунных факторов в этиологии синдромов липодистрофии.

*При поддержке гранта РНФ №17-75-30035 «Аутоиммунные эндокринопатии с полиорганными поражениями: геномные, постгеномные и метаболомные маркеры. Генетическое прогнозирование рисков, мониторинг, ранние предикторы, персонализированная коррекция и реабилитация».

\section{СПИСОК ЛИТЕРАТУРЫ | REFERENCES}

1. Chiquette E, Oral E, Garg A, et al. Estimating the prevalence of generalized and partial lipodystrophy: findings and challenges. Diabetes, Metabolic Syndrome and Obesity: Targets and Therapy. 2017;Volume 10:375-383. doi: 10.2147/dmso.s130810.

2. Brown RJ, Araujo-Vilar D, Cheung PT, et al. The Diagnosis and Management of Lipodystrophy Syndromes: A Multi-Society Practice Guideline. J. Clin. Endocr. Metab. 2016;101(12):4500-4511. doi: 10.1210/jc.2016-2466.

3. Agarwal AK, Garg A. A Novel Heterozygous Mutation in Peroxisome Proliferator-Activated Receptor- $\gamma$ Gene in a Patient with Familial Partial Lipodystrophy. J. Clin. Endocr. Metab. 2002;87(1):408-408. doi: 10.1210/jcem.87.1.8290.

4. Magré J, Delépine M, Khallouf E, et al. Identification of the gene altered in Berardinelli-Seip congenital lipodystrophy on chromosome 11q13. Nat. Genet. 2001;28(4):365-370. doi: 10.1038/ng585.

5. Garg A, Kircher M, del Campo M, et al. Whole exome sequencing identifies de novo heterozygousCAV1 mutations associated with a novel neonatal onset lipodystrophy syndrome. Am. J. Med. Genet. A. 2015;167(8):1796-1806. doi: 10.1002/ajmg.a.37115.

6. Kim CA, Delépine M, Boutet E, et al. Association of a Homozygous Nonsense Caveolin-1 Mutation with Berardinelli-Seip Congenital Lipodystrophy. J. Clin. Endocr. Metab. 2008;93(4):1129-1134. doi: 10.1210/jc.2007-1328.

7. Hayashi YK, Matsuda C, Ogawa M, et al. Human PTRF mutations cause secondary deficiency of caveolins resulting in muscular dystrophy with generalized lipodystrophy. J. Clin. Invest. 2009;119(9):26232633. doi: $10.1172 /$ jci38660.

8. Barsh GS, Rajab A, Straub V, et al. Fatal Cardiac Arrhythmia and LongQT Syndrome in a New Form of Congenital Generalized Lipodystrophy with Muscle Rippling (CGL4) Due to PTRF-CAVIN Mutations. PLoS Genet. 2010;6(3):e1000874. doi: 10.1371/journal.pgen.1000874.

9. Shastry S, Delgado MR, Dirik E, et al. Congenital generalized lipodystrophy, type 4 (CGL4) associated with myopathy due to novel PTRF mutations. Am. J. Med. Genet. A. 2010;152A(9):2245-2253. doi: 10.1002/ajmg.a.33578.

10. Herbst KL, Tannock LR, Deeb SS, et al. Kobberling Type of Familia Partial Lipodystrophy: An underrecognized syndrome. Diabetes Care. 2003;26(6):1819-1824. doi: 10.2337/diacare.26.6.1819.

11. Shackleton S, Lloyd DJ, Jackson SNJ, et al. LMNA, encoding lamin A/C, is mutated in partial lipodystrophy. Nat. Genet. 2000;24(2):153-156. doi: $10.1038 / 72807$

12. Barroso I, Gurnell M, Crowley VEF, et al. Dominant negative mutations in human PPARY associated with severe insulin resistance, diabetes mellitus and hypertension. Nature. 1999;402(6764):880-883. doi: $10.1038 / 47254$

13. Gandotra S, Le Dour C, Bottomley W, et al. Perilipin Deficiency and Autosomal Dominant Partial Lipodystrophy. N. Engl. J. Med. 2011;364(8):740-748. doi: 10.1056/NEJMoa1007487.

14. Rubio-Cabezas O, Puri V, Murano I, et al. Partial lipodystrophy and insulin resistant diabetes in a patient with a homozygous nonsense mutation inCIDEC. EMBO Mol. Med. 2009;1 (5):280-287. doi: 10.1002/ emmm.200900037.

15. Farhan SMK, Robinson JF, Mclntyre AD, et al. A Novel LIPE Nonsense Mutation Found Using Exome Sequencing in Siblings
With Late-Onset Familial Partial Lipodystrophy. Can. J. Cardiol. 2014;30(12):1649-1654. doi: 10.1016/j.cjca.2014.09.007.

16. Albert JS, Yerges-Armstrong LM, Horenstein RB, et al. Null Mutation in Hormone-Sensitive Lipase Gene and Risk of Type 2 Diabetes. N. Engl. J. Med. 2014;370(24):2307-2315. doi: 10.1056/NEJMoa1315496.

17. Semple RK, Sleigh A, Murgatroyd PR, et al. Postreceptor insulin resistance contributes to human dyslipidemia and hepatic steatosis. J. Clin. Invest. 2009. doi: 10.1172/jci37432.

18. De Sandre-Giovannoli A, Bernard R, Cau P, et al. Lamin a truncation in Hutchinson-Gilford progeria. Science. 2003;300(5628):2055-2055.

19. Donadille B, D'Anella P, Auclair M, et al. Partial lipodystrophy with severe insulin resistance and adult progeria Werner syndrome. Orphanet J. Rare Dis. 2013;8(1):106. doi: 10.1186/1750-1172-8-106.

20. Yu CE, Oshima J, Fu YH, et al. Positional Cloning of the Werner's Syndrome Gene. Science. 1996;272(5259):258-262. doi: 10.1126/science.272.5259.258

21. Goto M, Tanimoto K, Horiuchi Y, Sasazuki T. Family analysis of Werner's syndrome: A survey of 42 Japanese families with a review of the literature. Clin. Genet. 2008;19(1):8-15. doi: 10.1111/j.1399-0004.1981. tb00660.x.

22. Chen W, Zhou H, Saha P, et al. Molecular Mechanisms Underlying Fasting Modulated Liver Insulin Sensitivity and Metabolism in Male LipodystrophicBscl2/Seipin-Deficient Mice. Endocrinology. 2014;155(11):4215-4225. doi: 10.1210/en.2014-1292.

23. Csoka AB. Novel lamin $A / C$ gene (LMNA) mutations in atypical progeroid syndromes. J. Med. Genet. 2004;41 (4):304-308. doi: 10.1136/jmg.2003.015651.

24. Hussain I, Patni N, Ueda M, et al. A Novel Generalized Lipodystrophy-Associated Progeroid Syndrome Due to Recurrent Heterozygous LMNA p.T10l Mutation. J. Clin. Endocr. Metab. 2018;103(3):1005-1014. doi: 10.1210/jc.2017-02078

25. Novelli G, Muchir A, Sangiuolo F, et al. Mandibuloacral Dysplasia Is Caused by a Mutation in LMNA-Encoding Lamin A/C. The American Journal of Human Genetics. 2002;71 (2):426-431. doi: 10.1086/341908.

26. Agarwal AK, Simha V, Oral EA, et al. Phenotypic and Genetic Heterogeneity in Congenital Generalized Lipodystrophy. J. Clin. Endocr. Metab. 2003;88(10):4840-4847. doi: 10.1210/jc.2003-030855.

27. Simha V, Agarwal AK, Oral EA, et al. Genetic and Phenotypic Heterogeneity in Patients with Mandibuloacral Dysplasia-Associated Lipodystrophy. J. Clin. Endocr. Metab. 2003;88(6):2821-2824. doi: 10.1210/ jc.2002-021575.

28. Weedon MN, Ellard S, Prindle MJ, et al. An in-frame deletion at the polymerase active site of POLD1 causes a multisystem disorder with lipodystrophy. Nat. Genet. 2013;45(8):947-950. doi: 10.1038/ng.2670.

29. Lessel D, Vaz B, Halder S, et al. Mutations in SPRTN cause early onset hepatocellular carcinoma, genomic instability and progeroid features. Nat. Genet. 2014;46(11):1239-1244. doi: 10.1038/ng.3103.

30. Cabanillas R, Cadiñanos J, Villameytide JAF, et al. Néstor-Guillermo progeria syndrome: A novel premature aging condition with early onset and chronic development caused by BANF1 mutations. Am. J. Med. Genet. A. 2011;155(11):2617-2625. doi: 10.1002/ajmg.a.34249.

31. Graul-Neumann LM, Kienitz T, Robinson PN, et al. Marfan syndrome with neonatal progeroid syndrome-like lipodystrophy associated with a novel frameshift mutation at the $3^{\prime}$ terminus of the FBN1- 
gene. Am. J. Med. Genet. A. 2010;152A(11):2749-2755. doi: 10.1002/ ajmg.a.33690.

32. Masotti A, Uva P, Davis-Keppen L, et al. Keppen-Lubinsky Syndrome Is Caused by Mutations in the Inwardly Rectifying K+ Channel Encoded by KCNJ6. The American Journal of Human Genetics. 2015;96(2):295300. doi: 10.1016/j.ajhg.2014.12.011.

33. Agarwal AK, Xing C, DeMartino GN, et al. PSMB8 Encoding the $\beta 5$ i Proteasome Subunit Is Mutated in Joint Contractures, Muscle Atrophy, Microcytic Anemia, and Panniculitis-Induced Lipodystrophy Syndrome. The American Journal of Human Genetics. 2010;87(6):866-872. doi: 10.1016/j.ajhg.2010.10.031.
34. Savage DB, Semple RK, Clatworthy MR, et al. Complement Abnormalities in Acquired Lipodystrophy Revisited. J. Clin. Endocr. Metab. 2009;94(1):10-16. doi: 10.1210/jc.2008-1703.

35. Hegele RA, Cao H, Liu DM, et al. Sequencing of the Reannotated LMNB2 Gene Reveals Novel Mutations in Patients with Acquired Partial Lipodystrophy. The American Journal of Human Genetics. 2006:79(2):383-389. doi: 10.1086/505885.

36. Lupsa BC, Sachdev V, Lungu AO, et al. Cardiomyopathy in Congenital and Acquired Generalized Lipodystrophy. Medicine. 2010;89(4):245250. doi: 10.1097/MD.0b013e3181e9442f.

\section{ИНФОРМАЦИЯ ОБ АВТОРАХ [AUTHORS INFO]}

Соркина Екатерина Леонидовна, к.м.н. [Ekaterina L. Sorkina, PhD.]; адрес: Россия, 117036, Москва, улица Дм. Ульянова, д. 11 [address: 11 Dm. Ulyanova street, 117036 Moscow, Russia]; ORCID: https://orcid.org/0000-0001-7006-0664; eLibrary SPIN: 7777-0248; e-mail: ekaterina.sorkina@gmail.com.

Тюльпаков Анатолий Николаевич, д.м.н. [Anatoliy N.Tyulpakov, Sc.D.]; eLibrarySPIN: 8396-1798; e-mail: anatolytiulpakov@gmail.com

\section{ЦИТИРОВАТЬ:}

Соркина Е.Л., Тюльпаков А.Н.. Наследственные и приобретенные липодистрофии: Молекулярно-генетические и аутоиммунные механизмы. // Ожирение и метаболизм. - 2018. - Т.15. — №. 1 - C.39-42. doi: 10.14341/OMET2018139-42

\section{TO CITE THIS ARTICLE:}

Sorkina EL, Tyulpakov AN. Inherited and acquired lipodystrophies: molecular-genetic and autoimmune mechanisms. Obesity and metabolism. 2018;15(1):39-42. doi: 10.14341/OMET2018139-42 